\title{
Strongly interacting matter under rotation
}

\author{
Yin Jiang ${ }^{1, \star}$, Zi-Wei Lin ${ }^{2,3, \star \star}$, Xu-Guang Huang ${ }^{4,5, \star \star \star}$, and Jinfeng Liao ${ }^{6, \star \star \star \star}$ \\ ${ }^{1}$ School of Physics and Nuclear Energy Engineering, Beihang University, Beijing 100191, China. \\ ${ }^{2}$ Department of Physics, East Carolina University, Greenville, NC 27858, USA. \\ ${ }^{3}$ Key Laboratory of Quarks and Lepton Physics (MOE) and Institute of Particle Physics, Central China Nor- \\ mal University, Wuhan 430079, China. \\ ${ }^{4}$ Physics Department and Center for Particle Physics and Field Theory, Fudan University, Shanghai 200433, \\ China. \\ ${ }^{5}$ Key Laboratory of Nuclear Physics and Ion-beam Application (MOE), Fudan University, Shanghai 200433, \\ China. \\ ${ }^{6}$ Physics Department and Center for Exploration of Energy and Matter, Indiana University, 2401 N Milo B. \\ Sampson Lane, Bloomington, IN 47408, USA.
}

\begin{abstract}
The vorticity-driven effects are systematically studied in various aspects. With AMPT the distributions of vorticity has been investigated in heavy ion collisions with different collision parameters. Taking the rotational polarization effect into account a generic condensate suppression mechanism is discussed and quantitatively studied with NJL model. And in chiral restored phase the chiral vortical effects would generate a new collective mode, i.e. the chiral vortical wave. Using the rotating quark-gluon plasma in heavy ion collisions as a concrete example, we show the formation of induced flavor quadrupole in QGP and estimate the elliptic flow splitting effect for $\Lambda$ baryons.
\end{abstract}

\section{Introduction}

Pseudovector field-driven effects have attracted more and more interests recently [1-4]. As a parityeven and time-reversal-odd field, numbers of novel and non-trivial phenomena would be driven by it,such as chiral magnetic effect $[1,2,5,6]$. As another pseudovector field which is familiar to us, vorticity plays an important role in our daily life as well as in the high energy physics [7, 8]. In relativistic heavy ion collision experiments, the typical collision events are off-central and the created QCD matter will carry a nonzero angular momentum on the order of $10^{4} \hbar \sim 10^{5} \hbar$ with local angular velocity in the range $0.01 \sim 0.1 \mathrm{GeV}[9,10]$. There has also been impressive progress to study the rotating QCD matter using lattice gauge theory simulations [11]. Both first principle calculations and model studies suggest that vorticity would drive the phase transition [12] and anomalous transport [13-15]. In the following we will first simulate the collisions to extract the vorticity with AMPT model in the particle scenario [10]. With the results we would see the vorticity is so remarkable that we could

\footnotetext{
^e-mail: jiang_y@buaa.edu.cn

$\star \star$ e-mail: linz@ecu.edu

$\star \star \star$ e-mail: huangxuguang@fudan.edu.cn

$\star \star \star \star$ e-mail: liaoji@indiana.edu
} 
not simply neglect its impact on the phase transition and chiral transport. Hence we discuss the rotational polarization effects for quark pairings, which would change the chiral restoration and color superconductivity critical behaviors [12]. In chiral restored phase the vorticity would also induce two anomalous chiral transport relations for the left and right-handed quarks respectively $[13,14]$. Combining these two effects there is a new collective mode called chiral vortical wave(CVW) [15]. The CVW would reshuffle the final state hadrons' distributions so that provide a new contribution to the elliptic flow splitting of hadrons.

\section{Vorticity in heavy ion collisions}

As a transport model AMPT would output trajectories of all the partons in the phase space during collisions. For a large enough system after proper coarse-graining (e.g. like the fluid being made of many fluid cells), it can be considered as a continuous medium characterized by a series of locally defined quantities like momentum density, energy density and particle number density $\vec{p}(\vec{r}), \epsilon(\vec{r})$ and $n(\vec{r})$ respectively. Here we define the 3-velocity field as $\vec{v}(\vec{r})=\vec{p}(\vec{r}) / \epsilon(\vec{r})$. And the non-relativistic vorticity $\vec{\omega}=\nabla \times \vec{v}$ is adopted in this work. The detailed patterns of vorticity shown in [10] could be roughly understood by a radial flow profile $\vec{v}$ that can be well parameterized in the form $\vec{v}(\rho, \phi, \eta)=$ $\hat{e}_{\rho} v_{0}(\rho, \eta)\left[1+2 c_{2}(\rho, \eta) \cos 2 \phi\right]$, where $\hat{e}_{\rho}$ is the unit vector along the transverse radial direction, $\rho$ and $\phi$ are transverse radial and azimuthal coordinates. Noting that this flow profile has no global rotation. It suggests that, to extract the component of local vorticity that is truly associated with the global rotation, one needs to perform an average over the fireball. Upon such averaging, the background flow contributions to local vorticity would cancel out, and what remains can be attributed to the rotational motion. The averaged vorticity over the whole fireball depends on the weight we choose. In the

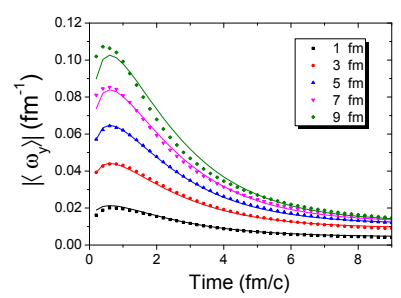

(a)

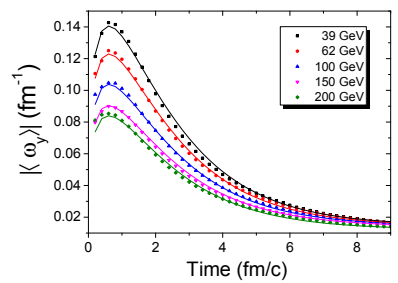

(b)

Figure 1. Averaged vorticity $\left\langle\omega_{y}\right\rangle$ from the AMPT model as a function of time at various impact parameter $b$ for fixed beam energy $\sqrt{s_{N N}}=200 \mathrm{GeV}$. (a). Averaged vorticity $\left\langle\omega_{y}\right\rangle$ from the AMPT model as a function of time at varied beam energy $\sqrt{s_{N N}}$ for fixed impact parameter $b=7 \mathrm{fm}$. (b).

following we choose $\mathcal{W}(\vec{r})=\rho^{2} \epsilon(\vec{r})$. Different weights dependence has also been investigated in [10]. The centrality dependence of $\left\langle\omega_{y}\right\rangle$ at given beam energy $\sqrt{s_{N N}}=200 \mathrm{GeV}$ : see Fig. 1(a). The $\left\langle\omega_{y}\right\rangle$ briefly increases with time which is most likely due to parton scatterings during the early stage (when the transverse radial expansion is not developed yet) that in certain way decrease the fluid moment of inertia. The averaged vorticity reaches peak value at an almost universal time around $1 \mathrm{fm} / \mathrm{c}$ and then follows a steady decrease with time. The decrease is due to the system's expansion which increases total moment of inertia at the price of reduced vorticity due to the constraint of constant angular momentum. The results also clearly demonstrate that the averaged vorticity increases from central to peripheral collisions: this trend is different from the angular momentum. Such difference again can be 
understood as follows: while the vorticity increases with $b$, the fluid moment of inertia (pertinent to rotation) in the fireball decreases with $b$, thus the angular momentum shows a non-monotonic behavior due to the two competing trends.

We next show the beam energy dependence of $\left\langle\omega_{y}\right\rangle$ at given impact parameter $b=7 \mathrm{fm}$ : see Fig. 1(b). Similar time evolution patterns are observed at all energies. We notice that the averaged vorticity increases with decreasing beam energy, in quite the opposite trend to the angular momentum. This may be understood as follows: with increasing beam energy, the fluid moment of inertia (pertinent to rotation) increases more rapidly than the decrease of vorticity, thus the total angular momentum is still increasing. We have numerically checked that this is indeed the case. And the trend has also been observed in STAR's results [9]

\section{Rotation-induced phase transition}

From the simulation we have already seen that the local vorticity can be several tens or one hundred of $\mathrm{MeV}$. Considering the polarization effect the quark energy shift should be $E \pm \vec{\omega} \cdot \vec{J}$ which behaves as a chemical potential. Therefore it would induce the non-negligible modification to the fermi surface of the quarks, which further affects the pairing probability. Theoretically it is more intuitive to study a fermionic system under a global rotation $\vec{\omega}$ to show the generic rotation-induce effect.

In this proceeding we only consider the $J=0$ pairing cases. For a pair of spin- $\frac{1}{2}$ fermions, there are different ways of forming a spin- 0 pairing state: either, the pair could have both nonzero orbital angular momentum $L$ and nonzero total spin $S$, with $L$ and $S$ being opposite thus resulting in total $J=0$; or the pair could have zero orbital angular momentum, and have opposite individual spin configurations for the two fermions. When such a system is under rotation, there will be a generic rotational suppression effect on the scalar pairing states. Intuitively this can be understood as follows. The global rotation, implying a nonzero macroscopic angular momentum of the whole system, will induce a rotational polarization effect which tends to "force" all microscopic angular momentum to be aligned with the global angular momentum. So for a pair of fermions, their relative orbital angular momentum $L$ as well as their individual spins would prefer to be parallel to the global angular momentum rather than to arrange themselves into a scalar state with zero angular momentum. This therefore leads to a generic suppression effect on the scalar pairing states. It also implies that pairing states with nonzero angular momentum could become more favorable. In the following, we quantitatively demonstrate this effect in the Nambu-Jona-Lasinio model for the chiral condensate (of quark-anti-quark pairing states with $L=S=1$ but $J=0$ ). And the diquark condensate (of quarkquark pairing state with $L=S=0$ ) case could be referred to [12].

In the chiral condensate case, the mean-field approximation gives the grand potential as

$$
\begin{aligned}
\Omega= & \int d^{3} \vec{r}\left\{\frac{(M-m)^{2}}{4 G}-\frac{N_{f} N_{c}}{16 \pi^{2}} \sum_{n} \int d k_{t}^{2} \int d k_{z} \times\left[J_{n}\left(k_{t} r\right)^{2}+J_{n}\left(k_{t} r\right)^{2}\right]\right. \\
& \left.\times T\left[\ln \left(1+e^{\left(\epsilon_{n}-\mu\right) / T}\right)+\ln \left(1+e^{-\left(\epsilon_{n}-\mu\right) / T}\right)+\ln \left(1+e^{\left(\epsilon_{n}+\mu\right) / T}\right)+\ln \left(1+e^{-\left(\epsilon_{n}+\mu\right) / T}\right)\right]\right\}
\end{aligned}
$$

In the above the mean-field quasiparticle dispersion $\epsilon_{n}$ is given by $\epsilon_{n}=\sqrt{k_{z}^{2}+k_{t}^{2}+M^{2}}-\left(n+\frac{1}{2}\right) \omega$. Note that because of the rotation around the z-axis, the $O(3)$ symmetry has been broken into $O(2)$ here. The mean-field chiral condensate (or equivalently the mass gap $M$ ) at given values of temperature $T$, chemical potential $\mu$ and rotation $\omega$, can then be determined from the usual gap equation through minimization the potential: $\frac{\delta \Omega}{\delta M(r)}=0$ and $\frac{\delta^{2} \Omega}{\delta M(r)^{2}}>0$. We will numerically solve the gap equation for the case of $N_{f}=2$ and $N_{c}=3$ and present the results below. For the parameters $G, G_{d}$ and a cutoff scale $\Lambda$ of this model, we choose the standard values (see e.g. [16]). 
The chiral restorations are observed in Fig 2(a) as the angular velocity increases at different temperatures. It is tempting to envision a new phase diagram of the chiral phase transition on the $T-\omega$ parameter space: see Fig. 2(b) (as computed from the present model). It features a chiral-symmetrybroken phase at low temperature and slow rotation while a chiral-symmetry-restored phase at high temperature and/or rapid rotation. A smooth crossover transition region at high $T$ and low $T$ and a first-order transition line at low $T$ and high $\omega$ are connected by a new critical end point. Given the present model parameters, this critical point is located at $T_{C E P}=0.020 \mathrm{GeV}$ and $\omega_{C E P}=0.644 \mathrm{GeV}$. As already discussed previously, the "rotational suppression" of the scalar condensate is a quite generic effect. It is conceivable that similar phase transition behaviors under rotation would also occur in other dynamical models for studying chiral condensate. In [12] we also studied the diquark

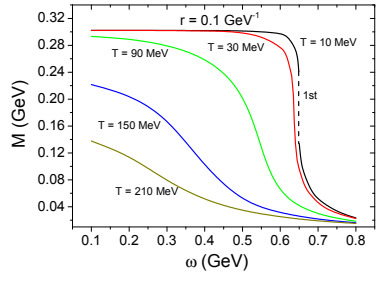

(a)

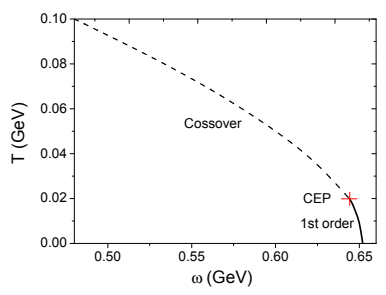

(b)

Figure 2. The mean-field mass gap $M$ (at radius $r=0.1 \mathrm{GeV}^{-1}$ ) as a function of $\omega$ for various fixed value of $T$ (a). The phase diagram on $T-\omega$ plane (b).

condensate case. As our analysis in above the rotational suppression would emerge again in that system. It should be mentioned that the rotational movement would always try to push matters far away from the center, which means it would be an inhomogeneous system. The radial distance-induced phase transition, which can not be smooth enough, is being studied self-consistently.

\section{Chiral vortical wave}

The CVW is the direct result of Chiral vortical effects

$$
\vec{J}_{V}=\frac{1}{\pi^{2}} \mu \mu_{5} \vec{\omega}, \quad \vec{J}_{A}=\left[\frac{1}{6} T^{2}+\frac{1}{2 \pi^{2}}\left(\mu^{2}+\mu_{5}^{2}\right)\right] \vec{\omega} .
$$

Using the chiral basis and considering the current conservation relations for the left and right-handed quark currents $\vec{J}_{L / R}=\frac{1}{2}\left(\vec{J}_{V} \mp \vec{J}_{A}\right)$, we would obtain the wave equations

$$
\partial_{t} n_{L / R}= \pm \frac{1}{4 \pi^{2}} \omega \partial_{x}\left(\mu_{L / R}^{2}\right)= \pm \frac{\omega \mu_{L / R}}{2 \pi^{2}} \partial_{x} \mu_{L / R},
$$

where the background vorticity is $\vec{\omega}=\omega \hat{x}$ and CVW speed is $V_{\Omega}=\frac{\mu_{0} \omega}{2 \pi^{2} \chi_{0}}$ in the linearized approximation. The $\chi_{\mu_{0}}$ is the susceptibility and $\mu_{0}$ the background chemical potential. Obviously the CVW will transport charges along or against the vorticity direction. The detailed process depends on the initial condition [15]. If the initial condition is a purely axial charge density fluctuation $F_{i}^{A}(x)$ at $t=0$, a charge dipole moment would be generated eventually with the size $(\delta n)_{t}^{V} \approx\left[-\partial_{x} F_{i}^{A}(x)\right] V_{\Omega} t$. On the other hand a purely vector charge density fluctuation $F_{i}^{V}(x)$ at $t=0$ would result a vector charge quadrupole $(\delta n)_{t}^{V} \approx F_{i}^{V}(x)+\left[\partial_{x}^{2} F_{i}^{V}(x) / 2\right]\left(V_{\Omega} t\right)^{2}$. 
To find possible experimental manifestation, we consider the rotating quark-gluon plasma created in off-central heavy ion collisions. In such a QGP, CVW occurs for each light flavors, e.g. $u, d$ quarks and possibly $s$ quarks as well. The global rotation points in the out-of-plane direction. Given initial fluctuations in their respective vector densities, each flavor develops its CVW along $\omega$. This transports flavor charges toward the two "tips" of the QGP fireball, thus leading to a quadrupole charge distribution on the transverse plane, which is shown in Fig. 3(a). In [15] we made the first estimate of such effect. The measurement may be done by the elliptic flow splitting of identified

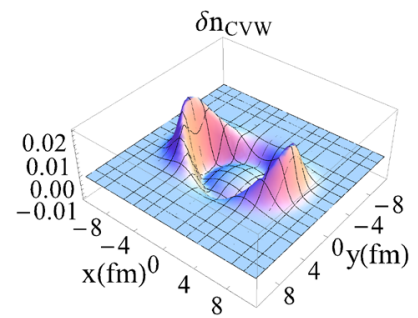

(a)

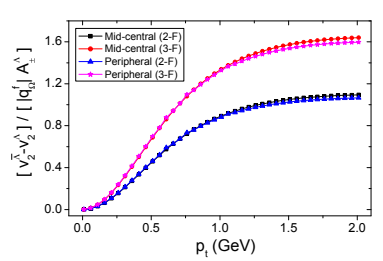

(b)

Figure 3. CVW-induced flavor charge density profile (a). Normalized $\bar{\Lambda}$ and $\Lambda$ elliptic flow splitting, $\left[v_{2}^{\bar{\Lambda}}-\right.$ $\left.v_{2}^{\Lambda}\right] /\left[\left|q_{\Omega}^{f}\right| A_{ \pm}^{\Lambda}\right]$, for symmetric 2-flavor(2-F) and 3-flavor(3-F) cases. The mid-central and the peripheral correspond to for $15-30 \%$ and $60-92 \%$ centrality class (see [17]) (b).

particles/anti-particles with nonzero baryonic charges. Flavor quadrupole implies more quarks on the out-of-plane tips of fireball and less on the in-plane equator, and therefore more baryons will be formed on the tips than on the equator. The stronger in-plane radial flow will thus translate the quadrupole into baryon/anti-baryon $v_{2}$ splitting. This mechanism is in analogy to the electric charge quadrupole induced by CMW [6]. To quantify such baryon flow spitting, one needs to combine quarks of varied flavors into hadrons. Suppose at the freeze-out, the flavor-wise chemical potential for quarks contains the CVW-induced quadrupole contribution $\delta \mu_{f} \propto 2 q_{\Omega}^{f} \cos \left(2 \phi_{s}\right)$ (with $\left.f=u, d, s\right)$. The corresponding chemical potential for a given type of hadron can be determined from its constituent quark content, e.g. for $\Lambda$ baryon $\delta \mu_{\Lambda} \propto 2\left(q_{\Omega}^{u}+q_{\Omega}^{d}+q_{\Omega}^{s}\right) \cos \left(2 \phi_{s}\right)$. We particularly propose to use $\Lambda$ baryon which is electric charge neutral thus unaffected by possible CMW effect. We then use the STAR blast-wave model [17] to compute the resulting differential flow splitting. As it is unclear how much the $s$ quark mass may reduce their chiral effects, we consider two extreme cases: a symmetric two-flavor (2-F) case $q_{\Omega}^{u}=q_{\Omega}^{d}=q_{\Omega}^{f}$ with $q_{\Omega}^{s}=0$, or a symmetric three-flavor (3-F) case $q_{\Omega}^{u}=$ $q_{\Omega}^{d}=q_{\Omega}^{s}=q_{\Omega}^{f}$. From Cooper-Frye scheme it is easy to see $\Delta v_{2}=v_{2}^{\bar{\Lambda}}-v_{2}^{\Lambda} \propto\left|q_{\Omega}^{f}\right| A_{ \pm}^{\Lambda}$ with $A_{ \pm}^{\Lambda}=$ $\left(N^{\Lambda}-N^{\bar{\Lambda}}\right) /\left(N^{\Lambda}+N^{\bar{\Lambda}}\right)$ the $\Lambda$-asymmetry that is directly related to background density $\mu_{0}$ (in analogy to a similar relation in CMW case [6]). The results for normalized flow splitting $\Delta v_{2} /\left[\left|q_{\Omega}^{f}\right| A_{ \pm}^{\Lambda}\right]$ are shown in Fig.3(b). Such effect in heavy ion collisions could be quantitatively investigated in the framework of anomalous fluid dynamics similarly to the recent development for the study of chiral magnetic effect [18].

\section{Summary}

In summary we systematically studied the vorticity-induced effects in various aspects. With AMPT it has been shown that the QGP not only carries large angular momentum and local vorticity, but also re- 
markable global rotation speed. Motivated by the large vorticity and taking the rotational polarization effect into account the spin zero condensate would be suppressed. As a generic suppression mechanism it may have non-negligible impacts in both QCD phase diagram and numbers of low energy systems. Finally in the chiral restored phase the vorticity would excite the chiral vortical wave. Using the rotating quark-gluon plasma in heavy ion collisions as a concrete example, we show the formation of induced flavor quadrupole in QGP and estimate the elliptic flow splitting effect for $\Lambda$ baryons. Such proposal could be tested with future experimental data.

Acknowledgments. This material is based upon work supported by the U.S. Department of Energy, Office of Science, Office of Nuclear Physics, within the framework of the Beam Energy Scan Theory (BEST) Topical Collaboration. The work is also supported in part by the NSF Grant No. PHY-1352368 (JL), by the DFG Collaborative Research Center "SFB 1225 (ISOQUANT)" (YJ), by the NSFC of China under Grants No. 11628508(ZWL), by Young 1000 Talents Program of China, NSFC with Grant No.11535012 and No.11675041, and the Scientific Research Foundation of State Education Ministry for Returned Scholars(XGH).

\section{References}

[1] D. Kharzeev and A. Zhitnitsky, Nucl. Phys. A 797, 67 (2007).

[2] D. E. Kharzeev, L. D. McLerran and H. J. Warringa, Nucl. Phys. A 803, 227 (2008).

[3] D. E. Kharzeev, J. Liao, S. A. Voloshin and G. Wang, Prog. Part. Nucl. Phys. 88, 1 (2016) doi:10.1016/j.ppnp.2016.01.001 [arXiv:1511.04050 [hep-ph]].

[4] J. Liao, Pramana 84, no. 5, 901 (2015) doi:10.1007/s12043-015-0984-x [arXiv:1401.2500 [hep$\mathrm{ph}]]$.

[5] D. E. Kharzeev and H. U. Yee, Phys. Rev. D 83, 085007 (2011) doi:10.1103/PhysRevD.83.085007 [arXiv:1012.6026 [hep-th]].

[6] Y. Burnier, D. E. Kharzeev, J. Liao and H. U. Yee, Phys. Rev. Lett. 107, 052303 (2011); arXiv:1208.2537 [hep-ph].

[7] L. P. Csernai, V. K. Magas, H. Stocker and D. D. Strottman, Phys. Rev. C 84, 024914 (2011) doi:10.1103/PhysRevC.84.024914 [arXiv:1101.3451 [nucl-th]].

[8] L. P. Csernai, D. D. Strottman and C. Anderlik, Phys. Rev. C 85, 054901 (2012) doi:10.1103/PhysRevC.85.054901 [arXiv:1112.4287 [nucl-th]].

[9] L. Adamczyk et al. [STAR Collaboration], Nature 548, 62 (2017) doi:10.1038/nature23004 [arXiv:1701.06657 [nucl-ex]].

[10] Y. Jiang, Z. W. Lin and J. Liao, Phys. Rev. C 94, no. 4, 044910 (2016) Erratum: [Phys. Rev. C 95, no. 4, 049904 (2017)] doi:10.1103/PhysRevC.94.044910, 10.1103/PhysRevC.95.049904 [arXiv:1602.06580 [hep-ph]].

[11] A. Yamamoto and Y. Hirono, Phys. Rev. Lett. 111, 081601 doi:10.1103/PhysRevLett.111.081601 [arXiv:1303.6292 [hep-lat]].

[12] Y. Jiang and J. Liao, Phys. Rev. Lett. 117, no. 19, 192302 (2016) doi:10.1103/PhysRevLett.117.192302 [arXiv:1606.03808 [hep-ph]].

[13] D. T. Son and P. Surowka, Phys. Rev. Lett. 103, 191601 (2009).

[14] D. E. Kharzeev and D. T. Son, Phys. Rev. Lett. 106, 062301 (2011).

[15] Y. Jiang, X. G. Huang and J. Liao, Phys. Rev. D 92, no. 7, 071501 (2015).

[16] S. P. Klevansky, Rev. Mod. Phys. 64, 649 (1992). doi:10.1103/RevModPhys.64.649

[17] F. Retiere and M. A. Lisa, Phys. Rev. C 70, 044907 (2004).

[18] Y. Jiang, S. Shi, Y. Yin and J. Liao, arXiv:1611.04586 [nucl-th]. 
(C) 2018. This work is licensed under http://creativecommons.org/licenses/by/4.0/ (the "License"). Notwithstanding the ProQuest Terms and conditions, you may use this content in accordance with the terms of the License. 